\title{
Applying the immobility theory to thoroughly solve the three Zeno's paradoxes
}

\author{
Ninh Khac Son \\ Date Performed: 12 February 2020
}

\begin{abstract}
- Applying the law of conservation of time to solve the Achilles and the tortoise paradox.

- Applying the smallest unit of time $T_{\min }$ in the universe to solve the $D i$ chotomy paradox.

- Applying the disappearing property of matter when moving to solve the Arrow paradox.
\end{abstract}

Key words: Zeno's paradoxes; Philosophy of motion; Foundations of motion.

\section{Achilles and the tortoise paradox}

1.1. Speaking of paradox

"In a race, the quickest runner can never over-take the slowest, since the pursuer must first reach the point whence the pursued started, so that the slower must always hold a lead." - Reference source Wikipedia.

Express the paradox in detail as follows:

Assume Achilles and the tortoise in a competition, when the referee starts to press time $T \in(0, \infty)$ :

- During the first time $T_{0}$, Achilles does not run, the tortoise runs at $v_{\text {tortoise }}=$ const_1

- During the next time $T_{1}$, Achilles runs at $v_{\text {Achilles }}=$ const_2 , the tortoise runs; the running speed of Achilles is greater than the speed of the tortoise.

$$
v_{\text {Achilles }}>v_{\text {tortoise }} \Leftrightarrow \text { const_2 }>\text { const_1 }
$$


- During the next time $T_{2}, T_{3} \ldots, T_{n}$, the velocity of Achilles and the turtle remain unchanged

$$
v_{\text {Achilles }}-v_{\text {tortoise }}=\Delta v=\text { const_3 }>0
$$

- Conclusion of paradox: Achilles does not catch the tortoise in every time $T=\sum_{i=0}^{n} T_{i} \in(0, \infty)$

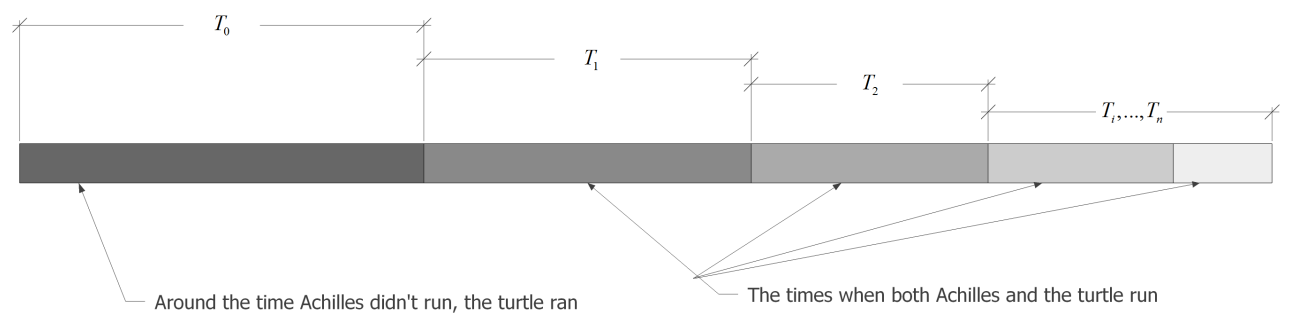

(Figure 1: Describe the time segments that Achilles and the tortoise achieve in the competition)

\subsection{Tools to solve}

Based on the immobility theory, the motion of the object in space is stated as follows: "All matter is in an intermittent motion for a time T large enough." - Reference source: Section one of the Immobility theory.

The equation of motion of the object according to the law of time conservation is as follows:

$$
t^{\prime}+t=T
$$

Where:

- $T$ is the intermittent movement time, the survey time of the object.

$$
T=\sum_{i=0}^{n} T_{i} \in(0, \infty)
$$

- $t^{\prime}$ is the total motion time of the object in time $T$.

$$
t^{\prime}=\sum_{i=0}^{n} t_{i}^{\prime}
$$


- $t$ is the total immobility time of the object in time T.

$$
t=\sum_{i=0}^{n} t_{i}
$$

The corresponding distances of moving objects with time periods $T_{0}, T_{1}, \ldots, T_{n}$ in the form:

$$
\begin{aligned}
& S_{i}=v_{i} * T_{i}=C * t_{i}^{\prime}+0 * t_{i}=C * t_{i}^{\prime} \\
& S=\sum_{i=0}^{n} S_{i}=C * \sum_{i=0}^{n} t_{i}^{\prime}=C * t^{\prime}
\end{aligned}
$$

Where:

- $S_{i}$ is the distance corresponding to $T_{i}$ or $t_{i}^{\prime}$ time.

- $C$ is the maximum velocity in the universe calculated according to the immobility theory.

\subsection{Methods of solving paradox}

The total motion time for Achilles and the tortoise in the competition is as follows:

$$
\begin{aligned}
& t_{\text {Achilles }}^{\prime}=t_{0 \_ \text {Achilles }}^{\prime}+\sum_{i=1}^{n} t_{i_{-} \text {Achilles }}^{\prime} \\
& t_{\text {Tortoise }}^{\prime}=t_{0 \_ \text {Tortoise }}^{\prime}+\sum_{i=1}^{n} t_{i_{-} \text {Tortoise }}^{\prime}
\end{aligned}
$$

Note: Because the distance quantities $S$, velocity $v$, and time $T$ are related, therefore we only need to analyze the motion time t' of the objects.

Because during $T_{0}$, Achilles does not run, so:

$$
t_{0 \_ \text {Achilles }}^{\prime}=0 \Rightarrow t_{\text {Achilles }}^{\prime}=\sum_{i=1}^{n} t_{i_{-} \text {Achilles }}^{\prime}
$$

And:

$$
t_{0 \_ \text {Tortoise }}^{\prime}=\text { const_4 }>0
$$


To summarize, the running competition of Achilles and the tortoise is represented by a system of equations:

$$
\left\{\begin{array}{l}
t_{\text {Achilles }}^{\prime}=\sum_{i=1}^{n} t_{i_{-} \text {Achilles }}^{\prime} \\
t_{\text {Tortoise }}^{\prime}=t_{0 \_ \text {Tortoise }}^{\prime}+\sum_{i=1}^{n} t_{i-\text { Tortoise }}^{\prime} \\
t_{0-\text { Tortoise }}^{\prime}=\text { const_4 }>0 \\
\sum_{i=1}^{n} t_{i_{-} \text {Achilles }}^{\prime}-\sum_{i=1}^{n} t_{i-T o r t o i s e}^{\prime}=\Delta t^{\prime}>0
\end{array}\right.
$$

The paradox only occurs if and only if $\Delta t^{\prime}<t_{0 \text {-Tortoise }}^{\prime}$ with $\forall T \in(0, \infty)$; But this is not possible. Because for every unit i for $T_{i}$, we always have:

$$
\begin{gathered}
v_{i \_ \text {Achilles }}>v_{i_{-} \text {Tortoise }} \\
\Rightarrow t_{i_{-} \text {Achilles }}>t_{i_{-} \text {Tortoise }} \\
\Rightarrow \lim _{T \rightarrow \infty} \Delta t^{\prime}=\infty>\text { const_4 } 4=t_{0_{-T o r t o i s e}}^{\prime}
\end{gathered}
$$

Therefore, there is always a time $\mathrm{T}$ that is large enough(limited) for Achilles to catch up to the tortoise and then pass.

\section{Comment:}

Zeno's methodology has a loophole: it only analyzes the movement of objects by the method of distance division $\mathrm{S}$, but ignores motion time t'.

Because time t' decides moving objects, this means that the value of $t$ ' is the cause of $S$ value - $S$ value is the result of $t$ ' value, so it is necessary to argue in the direction t' is proportional to $\mathrm{T}$ (the increase in $\mathrm{T}$ leads to $\mathrm{t}$ ' increase):

$$
t^{\prime}=\frac{v T}{C}
$$

and when $\mathrm{T}$ increases big enough then Achilles caught up to the tortoise: $\Delta t^{\prime}=t_{0 \_ \text {Tortoise }}^{\prime}$ 
But Zeno has argued the opposite, that is to consider that the distance $\mathrm{S}$ is the cause and analyzes the motion based solely on $\mathrm{S}$ and dividing the distance $S$ to infinity(mathematically pure), while the distance $S$ also have an indivisible $S_{\text {min }}$ limit (refer to the next section). This is an inverse argument that led to the problem-solving method that pushed the physical quantity $\mathrm{T}$ to infinity.

Currently, the paradoxical explanation is also based on pure mathematics based on convergent series:

$$
\sum_{i=1}^{\infty} \frac{1}{2^{i}}=1
$$

But this convergent series only solved mathematically, it did not solve philosophically, such as it did not explain why Achilles overtook the tortoise.

So if we use the convergent series (or geometry) to solve the paradox then we was trapped by him, because it is very possible that Zeno himself built this paradox based on his convergent series intuition.

Conclusion: The manuscript thoroughly solved both the philosophical and mathematical physics for the Achilles and the tortoise paradox.

\section{Dichotomy paradox}

\subsection{Speaking of paradox}

"That which is in locomotion must arrive at the half-way stage before it arrives at the goal."

Suppose Atalanta wishes to walk to the end of a path. Before she can get there, she must get halfway there. Before she can get halfway there, she must get a quarter of the way there. Before traveling a quarter, she must travel one-eighth; before an eighth, one-sixteenth; and so on.

The resulting sequence can be represented as:

$$
\left\{\ldots, \frac{1}{16}, \frac{1}{8}, \frac{1}{4}, \frac{1}{2}, 1\right\}
$$


(Figure 2: Before going all the way S, we need to make a first motion.)

This description requires one to complete an infinite number of tasks, which Zeno maintains is an impossibility.

This sequence also presents a second problem in that it contains no first distance to run, for any possible (finite) first distance could be divided in half, and hence would not be first after all. Hence, the trip cannot even begin.

Reference source Wikipedia.

\subsection{Tools to solve}

Based on section 5 of the immobility theory, the smallest unit of time $T_{\min }=$ const_5 in the universe is shown by the equation:

$$
T_{\min }=\frac{T}{n} * \sin (k) \cos (k)=\text { const }_{-} 5>0
$$

Where:

- $T_{\min }$ is the smallest time unit in the universe, $T_{\min }$ cannot be divided to be smaller.

- $n$ is the number of occurrences of particle in time T(frequency of particle occurrence).

- $k$ is the space compression ratio during the survey period $T$.

- T is total survey time.

Corresponding to $T_{\min }$ is a distance that $S_{\min }$ cannot be divided further in the movement of the object.

$$
S_{\min }=C * T_{\min }>0
$$

Reference source: Section 5 of the Immobility theory.

\subsection{Methods of solving paradox}

As a result of Equation 4, we see that the argument of the paradox states that there is no first distance to move is wrong, because $S_{\min }>0$ and it 
cannot be further subdivided.

Therefore, Atalanta can always start moving with $S_{\min }$ and the distance S(limited) that Atalanta wants to move:

$$
S=\sum S_{\min }
$$

Comment:

The paradox has relied too much on pure mathematics to say that everything can divide infinitely, thus creating a paradoxical conclusion.

But in fact the motion in nature is a bit different from pure math, which means that there is always the smallest $T_{\min }$ time to start moving with the $S_{\text {min }}$ distance.

Conclusion: This paradox is not quibble, but Zeno has not identified $T_{\min }$ and $S_{\min }$ and therefore he has not solved it.

\section{Arrow paradox}

\subsection{Speaking of paradox}

"If everything when it occupies an equal space is at rest, and if that which is in locomotion is always occupying such a space at any moment, the flying arrow is therefore motionless"

In the arrow paradox, Zeno states that for motion to occur, an object must change the position which it occupies. He gives an example of an arrow in flight. He states that in any one (duration-less) instant of time, the arrow is neither moving to where it is, nor to where it is not. It cannot move to where it is not, because no time elapses for it to move there; it cannot move to where it is, because it is already there. In other words, at every instant of time there is no motion occurring. If everything is motionless at every instant, and time is entirely composed of instants, then motion is impossible.

Reference source Wikipedia.

\subsection{Tools to solve}

Immobility theory describes all matter moving in space as follows: 
"With a time $\mathrm{T}$ large enough then matter always moves intermittently in space"

Time $\mathrm{T}$ is expressed in the equation:

$$
\begin{aligned}
& T=t_{1}^{\prime}+t_{1}+t_{2}^{\prime}+t_{2}+\ldots+t_{n}^{\prime}+t_{n} \\
& \Leftrightarrow T=\sum_{i=1}^{n} t_{i}^{\prime}+\sum_{i=1}^{n} t_{i}
\end{aligned}
$$

This means that the motion and the immobility of matter are always interwoven in an intermittent motion process with time $\mathrm{T}$ large enough.

Reference source: Section 1 of the Immobility theory.

\subsection{Methods of solving paradox}

Because the paradox argues that the arrow always occupies space, assuming that the arrow moves, it also occupies space, so it does not change its position, and the final conclusion of the paradox is that the arrow is immobile in space.

Here we should understand that Zeno considered motion to be a property of matter, thus creating a paradox. So we also assume motion is a property of matter to solve this paradox.

Zeno's argument for the motion of matter is contrary to the immobility theory, because the immobility theory has confirmed that matter moves if and only if it disappears for a period of time corresponding to $t_{i}^{\prime}$; and if it reappears - It is immobile for a period of time corresponding to $t_{i}$.

When matter disappears, it takes no place in space; matter only takes up space when it reappears.

Therefore when the arrow moves, it will disappear with time $t_{i}^{\prime}$; then it will reappear and remain motionless with $t_{i}$ time; At this time, its position had changed compared to its previous appearance. 
Example: The position of the arrow when it appears $i+1$ is a position away from the last time it appears(i) with a distance:

$$
\Delta S_{i+1}=S_{i+1}-S_{i}=C * t_{i+1}^{\prime}
$$

Comment:

Zeno's point of view is that the arrow always takes up space in every moment, so it cannot leave to another location, because wanting to move to another position forces the arrow to give up its previous position in space.

This is a good argument and it is not quibble, but Zeno does not yet know that matter(arrows) always have particle wave duality caused by the process disappears and appears continuously of the arrow in space, and it is also the disappearance of matter that changes its position in space.

Conclusion: The paradox has been thoroughly solved through the disappearing propertiy of matter.

\section{References}

[1] Zeno's paradoxes - Wikipedia

[2] Immobility theory - doi.org 